\title{
New Incremental Privacy-Preserving Clustering Protocols
}

\author{
Saeed Samet and Ali Miri
}

\begin{abstract}
We consider the problem of data clustering on streamed data, when the number of transactions is growing very quickly, or when data is distributed among several parties and their privacy is a concern. In this paper we present two new protocols for incremental privacy-preserving $k$-means clustering, which is a very popular data mining method, when data is distributed, horizontally or vertically, among multiple parties. At the end of each protocol, each party, without revealing its own private data, receives the final result of the clustering algorithm. Also, to improve efficiency, previous knowledge is used to incrementally update the centers and membership of each cluster.
\end{abstract}

Index Terms-Clustering, security and privacy-preserving, incremental algorithms, data mining and machine learning, distributed data structures.

\section{INTRODUCTION}

Data clustering is one of the most popular techniques in data mining, and is used to categorize information based on their similarities and differences. However, raw data is often distributed among several parties, each of which wants to preserve the privacy of its sensitive information. For instance, hospitals are not allowed to release their patients' data while they jointly need to use this information to categorize and cluster the data.

On the other hand, and in most applications, data is not static and changes frequently, making the use of standard, non-incremental protocols not suitable for these kinds of applications.

In this paper, we present new protocols for $k$-means privacy-preserving clustering that can not only be used with applications in which the data is distributed among several parties, but can also be applied incrementally on any streamed data.

The organization of the paper is as follows: in Section II, a brief introduction to $k$-means clustering is presented together with a brief overview of some of the existing work on incremental clustering. In Section III, we present two incremental privacy-preserving protocols, followed by conclusions and future work in Section IV.

\section{BACKGROUND}

\section{A. Non-Incremental Algorithms}

Privacy-preservation of sensitive information in data mining methods is a crucial issue in data communication and knowledge based systems. Many algorithms and protocols

Manuscript received February 1, 2013; revised April 15, 2013.

Saeed Samet is with the Faculty of Medicine, Memorial University of Newfoundland, St. John's, NL, Canada (e-mail: ssamet@mun.ca).

Ali Miri is with the Department of Computer Science, Ryerson University, Toronto, ON, Canada (e-mail: Ali.Miri@ryerson.ca). have been introduced for these methods such as Clustering [1]-[7], classification [8]-[18], and association rule mining [19]-[26].

The common purpose in these protocols is to maintain the privacy of the individuals and of the sensitive data of the parties involved, while each party derives some knowledge from the whole data set, owned by all the parties, without violating others' privacy.

The standard $k$-means clustering algorithm [27] is simple and relatively efficient. In this technique, at first $k$ arbitrary entities are produced as the initial means. Then each data entity (record or row) is assigned to the closest mean. In the next step, based on the entities in each cluster, centers are updated. The last two steps are repeated until the means remain unchanged or the difference between any new center and its corresponding previous value is less than a specific threshold.

Algorithm 1 [27] shows the complete algorithm for $k$-means clustering.

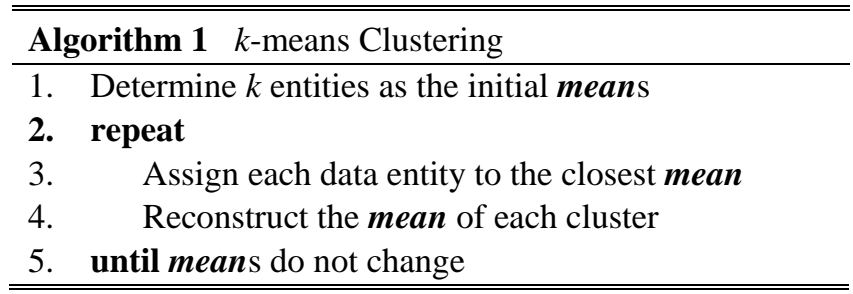

The distance function in the $k$-means clustering algorithm could be a common distance metrics such as Euclidean, Manhattan or Minkowski. Here we compute distance of two m-dimensional vectors $x$ and $y$ by:

$$
\sum_{i=1}^{m}\left(x_{i}-y_{i}\right)^{2}
$$

where $x_{i}$ and $y_{i}$ are the $i$-th elements of the vectors $X$ and $Y$ respectively. Also centroid, $\mu$, of a cluster containing $\left\{X_{1}, \ldots, X_{m}\right\}$ is

$$
\mu=\frac{X_{1}+\cdots+X_{m}}{m}
$$

However, by using this algorithm we have to re-scan the whole data inside a loop, until the loop condition is reached, i.e. means are not changed or the difference between previous means and new means are smaller than a specified threshold, and therefore it is not the best solution especially when we are faced with large databases, which is not unusual in real world applications.

\section{B. Incremental Algorithms}

Gupta and Grossman presented GenIc [28] which is a 
single pass algorithm for incremental clustering. In this algorithm, means are moved using a weighted sum of the existing means and the new item added each time.

The stream of data is divided into windows and each time a window is passed to the algorithm to assign its items to the means while the means are updated according to the incoming items. Actually, a centroid is fitted using its corresponding weight which is increased whenever an item is assigned to that centroid. Here is the GenIc algorithm adopted from [28]:

\section{1) Select Parameters.}

- Fix the number of centers, $k$.

- Fix the number of initial points, $m$.

- Fix the size of a generation $n$.

2) Initialize.

- Select $m$ points $c_{1}, \ldots, c_{m}$ to be the initial candidate centers.

- Assign a weight of $w_{i}=1$ to each center $c_{i}=1$.

3) Incremental Clustering. For each subsequent data point $p$ in the stream, do:

- Count $=$ Count +1 .

- Find the nearest candidate center $c_{i}$ to the point $p$.

- Move the nearest candidate center using the formula:

$$
c_{i}=\frac{w_{i} \times c_{i}+p}{w_{i}+1} .
$$

- Increment the corresponding weight: $w_{i}=w_{i}+1$.

- If Count $\equiv 0 \bmod n$, go to step 4 .

4) Generational Update of Clustering Centers. When Count equals $n, 2 n, 3 n, \ldots$, for every center $c_{i}$ in the list $L$ of centers, do:

- Calculate its probability of retention using the formula:

$$
p_{i}=\frac{w_{i}}{\sum_{i=1}^{m} w_{i}} .
$$

- Select a random number $\delta$ uniformly from $[0,1]$. If $p_{i}>\delta$ retain the center $c_{i}$ in the list $L$ of centers and use it in the next generation of $n$ points.

- If $p_{i}<\delta$, eliminate the center $c_{i}$ and select a new random point from the current generation to replace it as a center in the list $L$ of centers.

- Set the weight $w_{i}=1$.

- Go to step 3 and continue processing the input stream.

5) Calculate Final Clusters. The list $L$ contains the $m$ centers. These $m$ centers can be grouped into the final $k$ centers based on their Euclidean distances.

The greatest advantage of this algorithm is that it is single pass so each data item is examined only once and therefore the algorithm is very efficient compare to the standard $k$-means clustering algorithm. Also, it is very suitable and accurate for large databases.

The algorithm DIGNET [29] is another incremental clustering procedure. At each step, one data item is handled without iterative modification. Each center will be kept if the next coming data point belongs to that cluster and is eliminated otherwise. However the final results depend on the order of data items processed by the algorithm, and the algorithm can give poor results. It can, however, be used to generate initial centers for other methods.
Hartigan's leader algorithm [30] is another single-pass, incremental algorithm. In this algorithm, each data item is added to a center if it is close to it according to a specified threshold, otherwise a new center is created using that data item. There are also some other incremental algorithms used for hierarchical clustering, such as Charikar, et al. [31], which is not in the scope of our paper.

In our paper we use the GenIc incremental algorithm which was proposed by Gupta and Grossman [28]. However, we have made two types of modifications to this algorithm before using it in our secure protocols. First, in the 4th step of the GenIc algorithm, the threshold value $\delta$ will be randomly selected from $[0,1]$. If this value is very close to 1 in an iteration, one or more suitable means will be eliminated. On the other hand, if $\delta$ is very small, close to 0 , then many unsuitable means will be retained at the end of the iteration.

Either of these situations will decrease the accuracy of the final results at the end of each iteration, and thus, clustering based on the current clusters would not be as accurate as it should be, when compared to the regular $k$-means clustering method. As a simple example, suppose that in one iteration $\delta=0.99$ and none of the centroids is retained after the 4 th step, which is highly possible. This means that all of the previous data will be disregarded for the centroids from now on.

To prevent this problem, in our protocol we propose two changes. First, we set the range of possible values of $\delta$ to $[0.25,0.75]$ to make sure that no centroids with relatively high weight in an iteration will be eliminated, and also no controids with relatively low weight in an iteration will be retained. Second, we provide a reserved list to keep a certain number of eliminated centroids along with their weights.

Then, in each iteration, first we select the centroids by comparing the weights with $\delta$, and then each selected centroid's weight will be compared with the centroids' weights in the reserved list to select the centroid with the highest weight.

In this way, no centroid with relatively high weight would be deleted and no centroid with low weight would be retained. The best size of the proposed reserved list is the number of means or more. However, it could be optimized heuristically, and also based on the underlying data and disk space, although the reserved list will not need a large amount of space compared to the data.

We also modify the GenIc algorithm to apply to distributed data, and to preserve the privacy of the parties involved in the protocol.

\section{INCREMENTAL PRIVACY-PRESERVING $K$-MEANS Clustering}

In this section, we propose two new protocols for incremental privacy-preserving $k$-means clustering when data is horizontally or vertically partitioned among several parties.

\section{A. Protocol for Horizontally Partitioned Data}

In this case, each party owns some subset of records of the whole database and they jointly want to generate $k$ clusters for the database without revealing their own raw data to each other. For a database $D$, suppose each party $P_{i}(1 \leq i \leq m)$ 
owns a subset, $D_{i}$, of $D$ containing some entities such that

$$
\begin{gathered}
D_{i} \cap D_{j}=\emptyset \text { for any } 1 \leq i, j \leq m \\
\bigcup_{1 \leq i \leq m} D_{i}=D .
\end{gathered}
$$

The steps of the protocol are as follows:

1) Parties agree on: a threshold number, $\delta$ such that $0.25 \leq \delta \leq 0.75$ the number of means, $k$; and a chunk number, (the generation size), $n$.

2) Without loss of generality we order the parties involved in the protocol, $P_{1}, P_{2}, \ldots, P_{m}$.

3) $P_{1}$ randomly selects $k$ centers $C_{1}, C_{2}, \ldots, C_{k}$, assigns $w_{i}=1 \forall i \in\{1, \ldots, k\}$, and $s=1$.

4) $P_{s}$ selects $n$ items from its data $D_{s}$ to process. If $P_{s}$ has no more data, go to step 8.

5) For each selected item $A_{i}(1 \leq i \leq n)$ :

- $P_{s}$ finds the nearest centroid $C_{l}$ to $A_{i}$ i.e. for the distance function $d$ :

$$
d\left(A_{i}, C_{l}\right)<d\left(A_{i}, C_{j}\right) \forall j \in\{1, \ldots, k\} \text { and } j \neq l .
$$

- $C_{l}$ is adjusted using its corresponding weight, $w_{l}$, and item $A_{i}$ :

$$
C_{l}=\frac{w_{l} \times C_{l}+A_{i}}{w_{l}+1} .
$$

- $w_{l}=w_{l}+1$

6) For each weight $w_{i}(1 \leq i \leq k)$ :

- If $\frac{w_{i}}{\sum_{i=1}^{k} w_{i}} \geq \delta, C_{i}$ is retained as a center, otherwise it will be eliminated.

7) The list of selected centers will be merged and sorted with the centers in the reserved list. Then the $k$ highest ones will be selected as the retained centers. The unselected centers will be retained in the reserved list for the next iteration.

8) All $w_{i}$ s are set to 1 .

9) If $s=m$ then $s=1$, otherwise $s=s+1$.

10) If all the parties have processed their data, go to step 11.

11) All the centers are sent to party $P_{s}$, and go to step 4 .

12) The last party broadcasts the final centers to all parties.

13) Each party will assign its unassigned items to the closest centroid. These are the items whose centers have been eliminated in step 6 of the protocol.

Final centers will be stored by the parties as the initial centers for the next run of the protocol when data is added to the database.

- Security Analysis:

Inside the protocol, in each iteration some information is sent from one party to another party. We consider one such information transfer. Suppose $P_{i}$ sends the computed means $C_{1}, C_{2}, \ldots, C_{k}$ to $p_{i+1}$. For each mean $C_{l}$, we assume that $P_{i}$ had received $C_{l_{0}}$ from the previous party, $P_{i-1}$, and by continuing the algorithm, $C_{l}$ has been computed. Therefore we have:

$$
C_{l}=\frac{C_{l_{0}}+\sum_{r=1}^{n} A_{r}}{n+1} .
$$

Now $C_{l}$ is sent to $P_{i+1}$. However, $P_{i+1}$ has no information about $P_{i}$ 's items, $A_{r} \mathrm{~s}$, or the initial value of the centroid $C_{l_{0}}$.
Thus, by receiving $C_{l}, P_{i+1}$ is not able to get any detailed information about $P_{i}$ 's items, or the previous centroid, $C_{l_{0}}$. Even if two parties $P_{i-1}$ and $P_{i+1}$ collude by sending $C_{l_{0}}$ from $P_{i-1}$ to $P_{i+1}$, the second party will only know $\sum_{r=1}^{n} A_{r}$ of $P_{i}$ which is not enough to figure out individual items, $A_{r} \mathrm{~s}$. Note that we assume that $n$ is a large number.

\section{B. Protocol for Vertically Partitioned Data}

In this case, each party owns a subset of attributes from all records of the whole database. We denote the set of attributes owned by $P_{S}$ as

$$
A_{i, s}=\left\{a_{i, s_{1}}, a_{i, S_{2}}, \ldots, a_{i, s_{r}}\right\}
$$

For each centroid $C_{j}, P_{s}$ has the value of components corresponding to these attributes,

$$
\left\{C_{j, s_{1}}, C_{j, s_{2}}, \ldots, C_{j, s_{r}}\right\}
$$

The following are the steps of the protocol for this case:

1) Parties agree on: a threshold number, $\delta$ such that $0.25 \leq \delta \leq 0.75$; a number of means, $k$; and a chunk number, $n$.

2) Each party $P_{i}$ randomly selects its own part of the $k$ centers $C_{1}, C_{2}, \ldots, C_{k}$.

3) All $w_{i}$ s are publicly set to 1 .

4) Parties select $n$ unprocessed items from the whole database. Without loss of generality we have named them $A_{i}, A_{2}, \ldots, A_{n}$.

5) For each item $A_{i}(1 \leq i \leq n)$ :

- All parties run a privacy-preserving protocol to find the nearest center, say $C_{l}$, to that item. We use our proposed protocol presented in [5]. However, inside this protocol, we have used the two newer secure building blocks for secure addition and comparison proposed in [32], which are secure against collusion attacks as well.

- Each party adjusts its own part of $C_{l}$. For instance, suppose $P_{S}$ 's part of the item $A_{i}$ is

$$
A_{i, s}=\left\{a_{i, s_{1}}, a_{i, s_{2}}, \ldots, a_{i, s_{r}}\right\}
$$

and its part for the center $C_{l}$ is

$$
C_{l, s}=\left\{C_{l, s_{1}}, C_{l, s_{2}}, \ldots, C_{l, s_{r}}\right\}
$$

Thus, we have:

$$
C_{l, s}=\frac{w_{l} * C_{l, s}+A_{i, s}}{w_{l}+1} .
$$

- $w_{l}=w_{l}+1$

6) For each weight $w_{i}(1 \leq i \leq k)$ :

- If $\frac{\mathrm{w}_{\mathrm{i}}}{\sum_{\mathrm{i}=1}^{\mathrm{k}} \mathrm{w}_{\mathrm{i}}} \geq \delta, C_{i}$ is retained as a center, otherwise it will be eliminated.

7) The list of selected centers will be merged and sorted with the centers in the reserved list. Then the $k$ highest ones will be selected as the retained centers. The unselected centers will be retained in the reserved list for the next iteration. 
8) All $w_{i}$ s are set to 1 .

9) If there are more unprocessed data go to step 4.

10) Each party will assign its unassigned items to the closest centroid. These are the items whose centers have been eliminated in step 6 of the protocol.

At the end, each party stores its own portion of centers as the initial centers for the next run of the protocol. The privacy-preserving sub-protocol applied in the first part of step 5 uses secure comparison and secure sum. Refer to paper [5] for details. Security analysis of this protocol is the same as the previous one and is omitted here.

\section{CONCLUSIONS AND FUTURE WORK}

In this paper, two protocols for incremental privacy preserving $k$-means clustering are proposed. By using these protocols, parties are able to cluster their streamed data, while preserving their privacy. Due to the use of previous knowledge in an incremental algorithm, both communication and computational costs are decreased and protocols are more efficient than the normal ones. We use two secure sub-protocols, secure sum and secure comparison as building blocks inside our protocols. The design of incremental privacy-preserving protocols for other data mining and machine learning techniques, such as association rule mining and decision trees, could be some possible future work in this area.

\section{ACKNOWLEDGEMENT}

The work in this paper was supported in part by the Natural Sciences and Engineering Research Council of Canada (NSERC), and the Research \& Development Corporation of Newfoundland and Labrador (RDC).

\section{REFERENCES}

[1] S. Jha, L. Kruger, and P. McDaniel, "Privacy Preserving Clustering," in Proc. the 10th European conference on Research in Computer Security (ESORICS'05), Berlin, Heidelberg, 2005, pp. 397-417.

[2] J. Vaidya and C. Clifton, "Privacy-Preserving k-Means Clustering over Vertically Partitioned Data," in Proc. the ninth ACM SIGKDD international conference on Knowledge discovery and data mining, New York, NY, USA, 2003, pp. 206-215.

[3] G. Jagannathan and R. N. Wright, "Privacy-Preserving Distributed k-Means Clustering over Arbitrarily Partitioned Data," in Proc. the eleventh ACM SIGKDD international conference on Knowledge discovery in data mining, New York, NY, USA, 2005, pp. 593-599.

[4] G. Jagannathan, K. Pillaipakkamnatt, and R. N. Wright, "A New Privacy-Preserving Distributed k-Clustering Algorithm," in Proc. the Sixth SIAM International Conference on Data Mining, Bethesda, MD, USA, 2006.

[5] S. Samet, A. Miri, and L. Orozco-Barbosa, "Privacy Preserving k-Means Clustering in Multi-Party Environment," In Proc. the International Conference on Security and Cryptography (SECRYPT 2007), Barcelona, Spain, 2007, pp. 381-385.

[6] G. Jagannathan, K. Pillaipakkamnatt, R. N. Wright, and D. Umano, "Communication-Efficient Privacy-Preserving Clustering," Transactions on Data Privacy, vol. 3, issue 1, pp. 1-25, 2010.

[7] G. L. Aris Gkoulalas-Divanis, "Utility-guided Clustering-based Transaction Data Anonymization," Transactions on Data Privacy, vol. 5, issue 1, pp. 223-251, 2012.

[8] Y. Lindell and B. Pinkas, "Privacy Preserving Data Mining," Journal of Cryptology, pp. 36-54, 2000.

[9] R. Agrawal and R. Srikant, "Privacy-Preserving Data Mining," ACM Special Interest Group on Management of Data Conference, pp. 439-450, 2000.

[10] M.-J. Xiao, L.-S. Huang, Y.-L. Luo, and H. Shen, "Privacy Preserving ID3 Algorithm over Horizontally Partitioned Data," Parallel and
Distributed Computing, Applications and Technologies, pp. 239-243, 2005.

[11] W. Du and Z. Zhan, "Building Decision Tree Classifier on Private Data," in CRPITS'14: Proc. the IEEE international conference on Privacy, security and data mining, Darlinghurst, Australia, Australia, pp. 1-8, Australian Computer Society, Inc., 2002.

[12] J. Vaidya and C. Clifton, "Privacy-Preserving Decision Trees over Vertically Partitioned Data," Data and Application Security (DBSec), pp. 139-152, 2005.

[13] E. Suthampan and S. Maneewongvatana, "Privacy Preserving Decision Tree in Multi Party Environment," in Proc. the Second Asia conference on Asia Information Retrieval Technology, Jeju Island, Korea, 2005, pp. $727-732$.

[14] N. Zhang, S. Wang, and W. Zhao, "A New Scheme on PrivacyPreserving Data Classification," in Proc. the eleventh ACM SIGKDD international conference on Knowledge discovery in data mining, New York, NY, USA: ACM Press, 2005, pp. 374-383.

[15] S. Samet and A. Miri, " Privacy Preserving ID3 Using Gini Index over Horizontally Partitioned Data," in Proc. the 6th ACS/IEEE International Conference on Computer Systems and Applications (AICCSA-08), Doha, Qatar, March 2008, pp. 645-651.

[16] J. Zhan, "Privacy-preserving collaborative data mining," IEEE Computational Intelligent Magazine, pp. 31-41, May 2008.

[17] S. H. Xu and P. Sharkey, "Hongwei Tian, Weining Zhang. A Knowledge Model Sharing Based Approach to Privacy-Preserving Data Mining," Transactions on Data Privacy, vol. 5, issue 2, pp. 433-467, 2012.

[18] R. N. Wright, G. Jagannathan, and K. Pillaipakkamnatt, "A Practical Differentially Private Random Decision Tree Classifier," Transactions on Data Privacy, vol. 5, issue 1, pp. 273-295, 2012.

[19] C. Clifton, M. Kantarcioglu, and J. Vaidya, "Defining Privacy for Data Mining," National Science Foundation Workshop on Next Generation Data Mining, Baltimore, MD, pp. 126-133, November 2002.

[20] M. Kantarcioglu and C. Clifton, "Privacy-Preserving Distributed Mining of Association Rules on Horizontally Partitioned Data," IEEE Transactions on Knowledge and Data Engineering, vol. 16, issue 9, pp. 1026-1037, 2004.

[21] J. Z. Zhan, S. Matwin, and L. Chang, "Privacy-Preserving Collaborative Association Rule Mining," Data and Applications Security XIX, 19th Annual IFIP WG 11.3 Working Conference on Data and Applications Security, Storrs, CT, USA, pp. 153-165, 2005.

[22] J.-L. Wang, C.-F. Xu, and Y.-H. Pan, "An Incremental Algorithm for Mining Privacy-Preserving Frequent Itemsets," in Proc. 2006 International Conference on Machine Learning and Cybernetics, (Dalian, China), August 2006, pp. 1132-1137.

[23] Y. Duan, J. Canny, and J. Zhan, "Efficient Privacy-Preserving Association Rule Mining: P4P Style," IEEE Symposium on Computational Intelligence and Data Mining. CIDM 2007, Honolulu, Hawaii, USA, pp. $654-660,2007$.

[24] C.-M. Wu, Y.-F. Huang, and J.-Y. Chen, "Privacy Preserving Association Rules by Using Greedy Approach," WRI World Congress on Computer Science and Information Engineering, vol. 4, pp. 61-65, 2009.

[25] X. Ge, L. Yan, J. Zhu, and W. Shi, "Privacy-preserving distributed association rule mining based on the secret sharing technique," in Proc. 2nd International Conference on Software Engineering and Data Mining (SEDM), Chengdu, China, June 2010, pp. 345-350.

[26] Z. Yu-Quan, T. Yang, and C. Geng, "A Privacy Preserving Algorithm for Mining Distributed Association Rules," in Proc. International Conference on Computer and Management (CAMAN), Wuhan, China, May 2011, pp. 1-4.

[27] R. O. Duda, P. E. Hart, and D. G. Stork, Pattern Classification, 2nd ed., John Wiley, 2000.

[28] C. Gupta and R. L. Grossman, "GenIc: A Single-Pass Generalized Incremental Algorithm for Clustering," in Proc. the SIAM International Conference on Data Mining, 2004, pp. 137-153.

[29] S. C. A. Thomopoulos, D. K. Bougoulias, and C.-D. D. Wann, "Digent: An Unsupervised-Learning Clustering Algorithm for Clustering and Data Fusion," IEEE Transactions on Aerospace and Electronic Systems, vol. 31, issue 1, pp. 21-38, 1995.

[30] J. Hartigan, Clustering Algorithms, New York: John Wiley and Sons, 1975.

[31] M. Charikar, C. Chekuri, T. Feder, and R. Motwani, "Incremental Clustering and Dynamic Information Retrieval," Society of Industrial and Applied Mathematics (SIAM) Journal on Computing, vol. 33, issue 6, pp. 1417-1440, 2004.

[32] S. Samet and A. Miri, "Privacy-Preserving Back-Propagation and Extreme Learning Machine Algorithms," Journal of Data and Knowledge Engineering, vol. 79-80, pp. 40-61, 2012. 


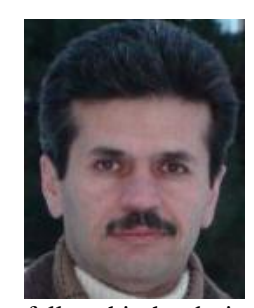

Saeed Samet has received his Ph.D. in Computer Science from the University of Ottawa and his thesis was in Privacy-Preserving Data Mining, in which he has proposed and designed several different protocols on privacy-preserving methods for standard data mining and machine learning techniques. He was then a Post-Doctoral Fellow at the e-Health Information Laboratory at the Children Hospital of Eastern Ontario Research Institute (CHEO-RI). During his post-doc fellowship he designed and developed several secure protocols for various health applications.

Currently, he is a member of the Faculty of Medicine, Memorial University of Newfoundland in Canada, as an Assistant Professor at the e-Health Research Unit at the discipline of Family Medicine, as well as being cross-appointment at the Department of Computer Science. He is the author and co-author of several peer-reviewed papers in international conferences and journals such as in JAMIA, DKE, and PLoS ONE, as well as a book, "Privacy Preserving Data Mining". His research interests include e-Health Security and Privacy, Health Informatics, Cryptography and Network Security, Secure Multi-party Computation, Privacy-Preserving in Data Mining and Machine Learning techniques, and Secret Sharing.

Dr. Samet has been TPC member, publication chair and publicity co-chair in several conferences, and currently is reviewer in several journals, such as JMIR, IJEHMC, eJHI, and IEEE-SMC. He has been awarded a grant from the Research $1 \&$ Development Corporation of Newfoundland and Labrador (RDC Ignite R\\&D), and he is a member of Society for Industrial and Applied Mathematics (SIAM).

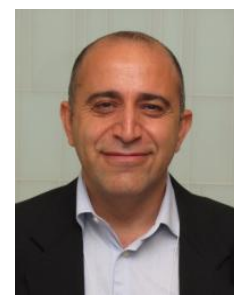

Ali Miri has been a full professor and associate chair at the School of Computer Science, Ryerson University, Toronto since 2009. He has also been with the Schoo of Electrical Engineering and Computer Science and the Department of Mathematics and Statistics of the University of Ottawa since 2001 as a Professor, and beginning in 2010 as an Adjunct Professor.

His research interests include computer networks, digital communication, and security and privacy technologies and their applications. He is the author and co-author of more than 150 peer-reviewed papers in international conferences and journals, 6 books and 4 patents.

Dr. Miri has also served on more than 60 organizing and technical program committees of international conferences and workshops, and has chaired a number of events including FPS 2012, SAC 2007 and 2011, IEEE WiMob 2010, PST 2010, CWIT 2009 and WSAN 2008. He has served on the editorial board the International Journal On Advances in Internet Technology of as a guest editor for the Journal of Ad Hoc and Sensor Wireless Networks, the Journal of Telecommunications Systems, and is currently serving on the editorial board of the Canadian Journal of Electrical and Computer Engineering (CJECE). He is a member of Professional Engineers Ontario, and a senior member of IEEE. 